\title{
Modélisation numérique de la formation d'un plasma induit par ablation laser UV (193 $\mathrm{nm})$
}

\author{
J.D. Parisse ${ }^{1,2}$, D.E. Zeitoun ${ }^{1}$, W. Marine ${ }^{2}$ et M.L. Sentis ${ }^{2}$ \\ 1 IUSTI, UMR 6595 du CNRS, Université de Provence, Technopôle de Château Gombert, \\ 5 rue Enrico Fermi, 13453 Marseille cedex 13, France \\ ${ }^{2}$ Groupe Interdisciplinaire Ablation Laser et Applications, LP3, FRE 2165, GPEC, UMR 6631, \\ Campus de Luminy, Case 917, 13288 Marseille cedex 9, France
}

\begin{abstract}
Résumé : Un modèle numérique permettant l'étude de l'interaction laser-matière en régime nanoseconde est développé. Le matériau considéré est du silicium et la longueur d'onde du laser dans le domaine spectrale de l'UV $(193 \mathrm{~nm})$. La modélisation, basée sur une approche monodimensionnelle instationnaire, se divise en deux parties. La première décrit l'interaction laser-cible, la seconde s'intéresse à l'interaction laser matière évaporée. Cette dernière est basée sur une description hydrodynamique de la matière évaporée tout en prenant en compte les processus physiques menant à la formation du plasma (absorption du rayonnement laser, ionisation du milieu ...). Les résultats issus du modèle numérique sont confrontés aux résultats expérimentaux extraits de la littérature.
\end{abstract}

\section{INTRODUCTION}

L'étude des différents phénomènes physiques intervenant lors de la formation d'un plasma généré par ablation laser est nécessaire tant pour l'amélioration des connaissances de tels processus que pour mieux maîtriser des procédés comme le dépôt de films ou la synthèse de nouveaux matériaux par ablation laser (PLD) ou des application de nettoyage, décontamination ou préparation de surfaces par laser. L'amélioration de nos connaissances passe bien entendu par l'étude expérimentale des processus d'ablation supportée par le développement de modèles numériques performants permettant la description de l'interaction laser-matière.

Le travail présenté dans cet article concerne l'étude détaillé de l'interaction laser matière uniquement lors de la durée de l'impulsion laser. La modélisation, basée sur une approche monodimensionnelle instationnaire, se divise en deux parties. La première décrit l'interaction laser-cible en résolvant les équations de la chaleur, de diffusion des porteurs libres et enfin de relaxation de l'énergie électronique [12]. La seconde partie traite de l'interaction laser matière évaporée. Cette dernière est basée sur une description hydrodynamique de la matière évaporée [3] tout en prenant en compte les processus physiques de formation du plasma [4] (absorption du rayonnement laser, ionisation du milieu ...).

\section{RESULTATS}

Le modèle développé permet l'étude de l'interaction laser-matière en régime picoseconde et nanoseconde. La cible utilisée pour nos simulation est du Silicium cristallin. L'impulsion laser considérée est celle d'un laser $\operatorname{ArF}(\lambda=193 \mathrm{~nm})$ ayant un forme gaussienne avec une largeur à mi-hauteur de $23 \mathrm{~ns}$.

Pour valider le modèle numérique les résultats expérimentaux publiés par Sanchez et a1. [5] ont été utilisés. Les conditions d'irradiation sont une densité d'énergie de $4 \mathrm{~J} / \mathrm{cm}^{2}$ pour une pression environnante de 1 atmosphère. La profondeur ablatée mesurée par Sanchez et al. est de l'ordre de $80 \mathrm{~nm}$, alors que a profondeur ablatée déterminée à l'aide du code de calcul est de $70 \mathrm{~nm}$. On constate donc un bon accord entre valeur expérimentale et valeur théorique.

La figure 1, montre l'évolution spatiale de la température de la vapeur $(\mathrm{T})$ et de la température électronique ( $\mathrm{Te}$ ) en fin d'impulsion laser pour une pression ambiante $(\mathrm{Pb})$ de 1 bar d'Hélium. On constate ainsi qu'en fin d'irradiation du matériau, l'équilibre thermique est pratiquement atteint $(T=\mathrm{Te})$. Les températures sont de l'ordre de 1 à $2 \mathrm{eV}$ au voisinage de la surface. 


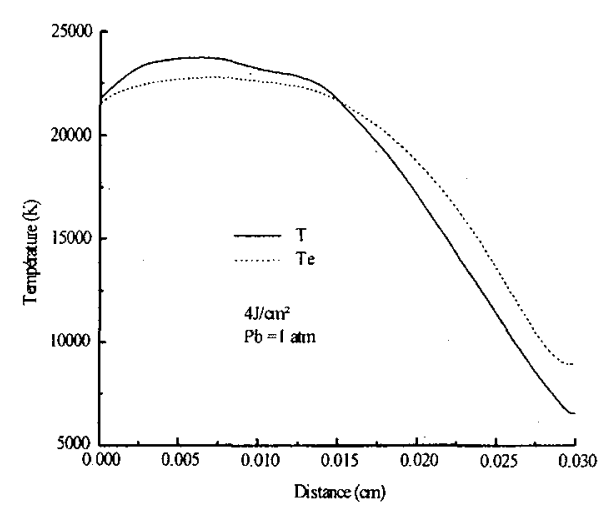

Figure 1 : Champs des diffërentes densités à la fin de l'impulsion laser.

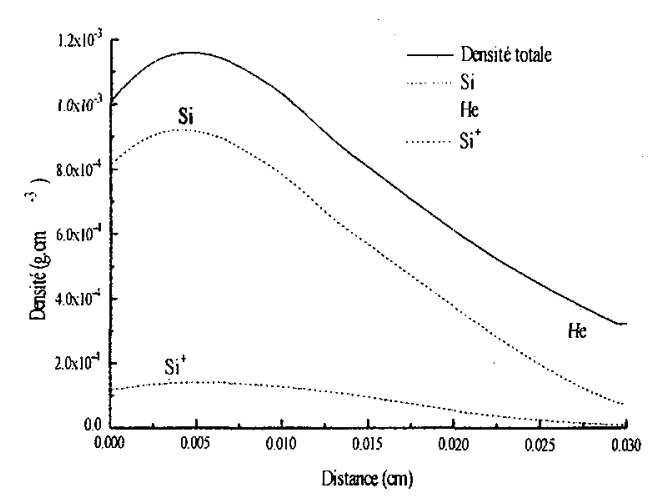

Figure 2 : Distribution spatialeen fn d'inpuslion des diffrentes densités

La figure 2, représente l'évolution spatiale des différentes densités également en fin de durée d'impulsion laser. Le taux d'ionisation moyen est alors d'environ $15 \%$, ceci expliquant les valeurs élevées des températures car avec un tel degré d'ionisation les processus de chauffage par effet Bremstralhung Inverse sont non négligeables [3]. Sur cette figure nous pouvons aussi observer la position du choc se propageant dans le gaz ambiant (He), ainsi que la détente de ce dernier, cette dernière correspond au maximum de densité du gaz ambiant c'est à ire à environ $30 \mu \mathrm{m}$ de la surface.

\section{CONCLUSION}

Ces travaux ont permis le développement d'un code de calcul fiable permettant de décrire la formation d'un plasma lors de l'irradiation d'un semi-conducteur avec un laser UV en régime nanoseconde. Ce modèle permet de donner en fin d'impulsion laser les grandeurs caractéristiques du plasma qui serviront par la suite de conditions initiales à un modèle d'expansion développé antérieurement [6.]

\section{Références}

[1] J.D. Parisse, D. Zeitoun, W. Marine et M. Sentis « Etude Numérique de l'interaction laser matière en régime pico et nanoseconde ", Journal de physique IV France 9 (1999)

[2] H.M. Van Driel « Kinetics of high-density plasmas generated in Si by 1.06 and $053 \mu \mathrm{m}$ picosecond laser pulses », Physical Review B 1987, Volume 35, Number 15

[3] A. Vertes et al. «Hydrodynamics modelling of laser plasma ionisation processes » Internationnal Journal of Mass Spectrometry and Ion processes 1989

[4] D.I. Rosen et al. "Coupling of plused $0.35 \mu \mathrm{m}$ laser radiation to aluminiun alloys », Journal of Applied Physics 1982, Volume 4

[5] F. Sanchez, J.L. Morenza, R. Aguiar, J.C. Delgado, and M. Varela « Whiskerlike structure growth on silicon exposed to ArF excimer laser irradiation », App. Phys. Lett. 69 (5), 29 July 1996620

[6] H.C. Le « Etude Numérique et expérimentale de l'expansion du plasma produit par ablation laser sous atmosphère neutre », Thèse de l'Université de Provence Juin 1997 